PHYS. MED. BIOL., 1972, VOL. 17, NO. 2, 232-240

\title{
An Algorithm for LET-analysis
}

\author{
A. M. KELLERER, PH.D. \\ Department of Radiology, Radiological Research Laboratories, \\ College of Physicians and Surgeons, \\ Columbia University, New York, NY 10032, U.S.A.
}

Received 27 May 1971

\begin{abstract}
An algorithm for the derivation of LET-distributions from pulse-height spectra obtained with proportional counters is described. The method is based on Fourier transformation; it is applicable to spherical as well as non-spherical proportional counters. The relation between the energy mean, $\bar{L}_{D}$, of LET and the energy mean, $\bar{y}_{D}$, of the lineal energy density is given.
\end{abstract}

\section{Introduction}

Tissue equivalent proportional counters are frequently used for the analysis of LET-distributions in neutron fields (Rossi and Rosenzweig 1955; Wilson and Field 1970). The method is limited by the fact that the experimentally observed pulse height distributions are the result of various random factors which cannot in general be easily separated. These limitations of the LETanalysis with proportional counters have been one of the starting points of microdosimetry when Rossi and his co-workers realized that the observed distributions are more closely related to the biological effectiveness of a given type of radiation than the distribution of linear energy transfer. Linear energy transfer is only a mean value of the rate of energy loss of a charged particle along its track, while the observed pulse height distributions reflect the actual variations of energy deposition in the irradiated tissue.

There are, however, cases where one needs an approximate overall evaluation of radiation quality for the purpose of comparing different types of radiation. In these cases, and this refers particularly to radiotherapy and to radiation protection applications, LET-distributions offer a convenient frame of reference. It is for this reason that the present paper re-evaluates the algorithm used in the LET-analysis.

The established method of LET analysis (Rossi and Rosenzweig 1955) is based on the assumption that energy loss straggling of the heavy charged particle, radial extension of its $\delta$-ray halo and finite length of its track can be neglected. It is assumed that a charged particle traverses the sensitive region of the counter without change of its LET, and that the energy deposited is the product of the LET and of the chord length in the region. These assumptions are tenable only under the two conditions: (i) that the neutron energies are high enough so that most of the recoil tracks are long compared with the dimensions of the region and (ii) that the stopping power of the particles and the diameter of the region are large enough that energy loss straggling and the efflux and influx of $\delta$-rays can be neglected. Strictly speaking these conditions 
are mutually exclusive. It will not be the object of this paper to examine the deviations from the idealized assumptions, or to discuss methods to account for these deviations. Instead it will be postulated that the measurements are performed in a range of neutron or heavy charged particle energies and site diameters for which the two conditions mentioned above are approximately fulfilled. Under these assumptions, that is within the framework of the conventional approximation, an algorithm will be described which has certain advantages over the established method.

\section{Formulation of the problem and discussion of the conventional solution}

Under the simplifications mentioned above the problem of the LET-analysis can be formulated in the following way. A region, $U$, is randomly traversed by charged particles. The distribution of chord length, $l$, resulting in the random traversal is $g(l)$. The distribution of LET is $t(L)$. The energy, $E$, deposited in $\mathrm{U}$ during a traversal is the product of the two independent random variables $l$ and $L$. The distribution, $f(E)$, of $E$ is experimentally determined; the chordlength distribution, $g(l)$, is known; the LET-distribution, $t(L)$, has to be derived.

Let the sum distributions belonging to the densities $f(E), g(l)$ and $t(L)$ be denoted by $F(E), G(l)$ and $T(L)$. For example $F(E)$ is the probability that the energy deposited in a passage is equal to or less than $E$. Then one obtains

$$
F(E)=\int_{0}^{\infty} t(L) G(E / L) \mathrm{d} L
$$

and, therefore,

$$
f(E)=\frac{\mathrm{d} F(E)}{\mathrm{d} E}=\int_{0}^{\infty} t(L) g(E / L) \frac{\mathrm{d} L}{L} .
$$

In the special case of a sphere of diameter $d$ one has the chord-length distribution:

$$
g(l)=2 l / d^{2} \quad l \leqslant d
$$

and, therefore,

$$
f(E)=\frac{2 E}{d^{2}} \int_{L=E / d}^{\infty} t(L) L^{-2} \mathrm{~d} L .
$$

By differentiation one obtains

$$
\frac{1}{E} \frac{\mathrm{d} f(E)}{\mathrm{d} E}-\frac{f(E)}{E^{2}}=-\frac{2 t(E / d)}{d E^{2}}
$$

and, therefore,

$$
t(L)=\frac{d}{2}\left\{-E \frac{\mathrm{d} f(E)}{\mathrm{d} E}+f(E)\right\} \quad \text { with } E=L d .
$$


This equation corresponds to the formula of Rossi and Rosenzweig (1955) which is commonly applied to derive LET-spectra from pulse height distributions obtained with spherical proportional counters. The method is limited by the fact that it applies to the sphere and not in general to other chordlength distributions. Nor can it be applied in the case where one deals with sensitive regions of different diameters; the latter would for instance be the case if measurements were taken from an assemblage of microscopic spherical scintillators.

It is also a certain restriction that application of eqn (6) necessitates numerical smoothing of the experimental distribution $f(E)$ in order to convert this spectrum into a differentiable function.

In the following section an alternative technique of LET-analysis will be discussed which is applicable to arbitrary chord-length distributions and which makes it unnecessary to convert the experimental data into a differentiable function.

\section{Solution of the problem by Fourier transformation}

$E$ is the product of two independent random variables $L$ and $l$. It is, however, more convenient to deal with the sum of two random variables than with their product. Therefore, instead of the variables $E, L$ and $l$ their logarithms will be considered. The new variables and their distributions will be designated by Greek letters :

$$
\varepsilon=\ln E, \quad \Lambda=\ln L \quad \text { and } \quad \lambda=\ln l .
$$

The probability distributions of the logarithms can be expressed in terms of the original variables and their distributions:

$$
\phi(\varepsilon)=\frac{\mathrm{d} F(E)}{\mathrm{d} \ln E}=E \frac{\mathrm{d} F(E)}{\mathrm{d} E}=E f(E)
$$

and in an analogous way

$$
\begin{gathered}
\tau(\Lambda)=\frac{\mathrm{d} T(L)}{\mathrm{d} \ln L}=\operatorname{Lt}(L) \\
\gamma(\lambda)=\frac{\mathrm{d} G(l)}{\mathrm{d} \ln l}=\lg (l)
\end{gathered}
$$

$\varepsilon$ is the sum of the two random variables $\lambda$ and $\Lambda$, and the distribution of $\varepsilon$ is equal to the convolution integral of the distributions of $\lambda$ and $\Lambda$ :

$$
\phi(\varepsilon)=\int_{-\infty}^{\infty} \tau(\varepsilon-\lambda) \gamma(\lambda) \mathrm{d} \lambda
$$

It is not necessary to evaluate this integral equation in order to obtain $\tau(\Lambda)$ from $\phi(\varepsilon)$ and $\gamma(\lambda)$. Instead one can use the convolution theorem (see e.g. Feller 1950). According to this theorem the Fourier transform, or in the 
language of probability theory the characteristic function, of the distribution of $\varepsilon$ is equal to the product of the Fourier transforms of the distributions of $\lambda$ and $\Lambda$. Accordingly one has

$$
\tau^{*}(x)=\phi^{*}(x) / \gamma^{*}(x)
$$

if $\tau^{*}(x), \phi^{*}(x)$ and $\gamma^{*}(x)$ are the Fourier transforms of the distributions $\tau(\Lambda)$, $\phi(\varepsilon)$ and $\gamma(\lambda)$. By a reverse Fourier transform applied to $\tau^{*}(x)$ one obtains $\tau(\Lambda)$ and thereby $t(L)$.

The fast Fourier transform algorithm of Cooley and Tukey (1965) is now widely available even in versions for small computers. The numerical execution of the solution described above therefore presents no practical difficulties. $\dagger$

A numerical example can be used to illustrate the different steps involved in the computation. The curve in fig. $1(a)$ represents a pulse-height distribution, $\phi(\varepsilon)=E f(E)$, obtained with a spherical proportional counter exposed to a field of 3.7 MeV neutrons plus a $\gamma$-ray component (Biavati, Rossi and Boer 1965). The curve in fig. 1 (b) represents the chord-length distribution, $\lg (l)$, in the sphere.
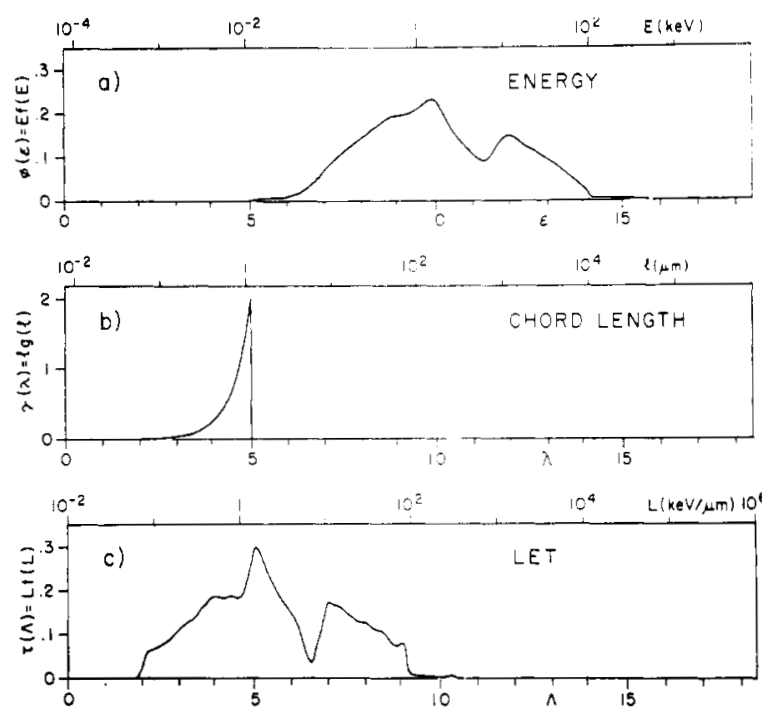

Fig. 1. Example for the interrelation between energy deposition spectrum, chord-length distribution, and LET-distribution. The energy deposition spectrum (a) has been obtained in a field of $3.7 \mathrm{MeV}$ neutrons with a spherical proportional counter of an equivalent diameter of $1.16 \mu \mathrm{m}$. The solution (c) is such that its convolution with the chord-length distribution (b) is equal to (a).

In the numerical evaluation the functions are represented by arrays which contain the function values on an equidistant point grid over the intervals depicted in fig. 1. The distribution $\phi(\varepsilon)$, for example, is represented by the

$\dagger$ A computer code of the solution described in this paper is available in FORTRAN IV. It accepts two input arrays $f(E)$ and $g(l)$ and derives $t(L)$, its sum distribution and its moments. The program uses the Cooley-Tukey fast Fourier transform in the version of the I.B.M. SHARE-library. 
array $\phi\left(\varepsilon_{1}\right), \mathrm{i}=0,1, \ldots, N-1$. The number, $N$, of points in the array can be varied, but it has to be a power of 2 for the fast Fourier transform algorithm of Cooley and Tukey. For this algorithm the computing times are proportional to $N \ln N$ and not to $N^{2}$ as would normally be the case. Therefore, very high values of $N$ may be chosen; typically, however, a value of $N=1024$ is sufficient. The Fourier transform is calculated as

$$
\phi^{*}\left(x_{k}\right)=\sum_{i=0}^{N-1} \exp (2 \pi i k / N) \phi\left(\varepsilon_{k}\right) \quad k=0,1, \ldots, N-1
$$

This amounts to treating the distribution $\phi(\varepsilon)$ as periodic over the full interval shown in fig. 1 ; the assumption does not introduce errors as long as the interval is large compared with the total width of the distribution. Relations corresponding to eqn (13) hold for the functions $\gamma^{*}(x)$ and $\tau^{*}(x)$. The reference interval, that is the interval of periodicity, has to be the same for the variables $\varepsilon, \lambda$ and $\Lambda$. In terms of the original variables this means that $E, l$ and $L$ vary over the same factor; in the example of fig. 1 this is a factor of $10^{8}$. A change in the units of $E, l$ and $L$ amounts to a shift of the distributions in fig. 1. A suitable choice of the units brings the distributions into the inside of the interval; this is not essential but a matter of convenience.

According to eqn (13) the component $\phi^{*}\left(x_{k}\right)$ in the transformed array belongs to the frequency $k$ in the full interval. In order to obtain the array $\tau^{*}\left(x_{k}\right)$, which is the transform of the LET-distribution, one divides the two complex arrays $\phi^{*}\left(x_{k}\right)$ and $\gamma^{*}\left(x_{k}\right)$ component by component. Because the original arrays are real valued functions the $k$ th and the $(N-k)$ th component of the transforms are conjugated complex. This can easily be seen from eqn (13).

The solution $\tau(\Lambda)=L t(L)$ which is obtained by a backwards transformation from $\tau^{*}(x)$ :

$$
\tau\left(\Lambda_{i}\right)=\sum_{k=0}^{N-1} \exp (-2 \pi k i / N) \tau^{*}\left(x_{k}\right) \quad i=0,1, \ldots, N-1
$$

is plotted in fig. $1(c)$. It is not practicable to plot the characteristic functions because they vary too rapidly with $x$.

It should be noted that the assumptions underlying the LET-analysis are not valid in the region of low LET, and that, therefore, the initial part of the function $L t(L)$, which corresponds to the $\gamma$-ray component of the radiation field, is meaningless. It should further be noted that one may in certain cases obtain negative values in the solution. This then means that there is no distribution which, folded into the chord-length distribution, yields the experimentally observed spectrum. The reason can be experimental inaccuracies or inapplicability of the simplifying assumptions discussed in section 1.

In the preceding discussion the multiplicative relation between the three random variables $L, l$ and $E$ has been reduced to an additive relation by transition to the logarithms. This transition made it possible to apply the 
Fourier transformation; in the same way one may choose the Laplace transform. It should be noted that the transition to the logarithms and their distributions could be avoided if instead of the characteristic functions (Fourier transforms) one were to use the so-called moment generating functions. This reflects the fact that the moment generating function of a distribution is essentially equal to the characteristic function of the distribution of the logarithm of the random variable. Transition to the logarithm has, however, great advantage because the logarithms cover a much narrower range of values than the original variables $L$ and $E$. Fourier analysis has previously been used for the solution of the energy straggling problem (Kellerer 1970). In this case one deals with the variable $E$ and not its logarithm. This, and the fact that microdosimetric distributions, as in the example of fig. 1, extend over many orders of magnitude, can lead to excessive array sizes in the numerical computations. In the LET-analysis no such problems arise because the logarithmic distributions are always sufficiently narrow.

\section{Evaluation of the moments of the distribution}

The energy, $E$, deposited in a passage is the product of the two statistically independent random variables $l$ and $L$. Therefore, the moments of $E$ are the product of the moments of $l$ and $L$. By using eqn (2) one can show this as follows :

$$
\begin{aligned}
\overline{E^{n}} & =\int_{0}^{\infty} E^{n} f(E) \mathrm{d} E \\
& =\int_{0}^{\infty} E^{n} \int_{0}^{\infty} t(L) g(E / L) \frac{\mathrm{d} L}{L} \mathrm{~d} E \\
& =\int_{0}^{\infty} t(L) L^{n}\left\{\int_{0}^{\infty}\left(\frac{E}{L}\right)^{n} g\left(\frac{E}{L}\right) d \frac{E}{L}\right\} \mathrm{d} L \\
& =\overline{l^{n}} \int_{0}^{\infty} t(L) L^{n} \mathrm{~d} L=\overline{l^{n}} \overline{L^{n}} .
\end{aligned}
$$

In microdosimetry the lineal energy density, $y$, is frequently used instead of the variable $E$. This quantity is defined (ICRU 1971) by

$$
y=E / \bar{l}
$$

where $l$ is the mean chord length of the region of interest. The quantity $y$ is the microdosimetric analogue of the linear energy transfer $L$. According to eqn (14) one has

$$
\overline{y^{n}}=\overline{L^{n}} \overline{l^{n}} / \bar{l}^{n}
$$

Specifically, one has the mean values $: \dagger$

$$
\bar{y}=\bar{L}
$$

$\dagger$ These mean values, that is the first moments of the distributions $f(y)$ and $t(L)$, are usually termed frequency mean, $\bar{y}_{F}$, of $y$ and track mean, $\bar{L}_{T}$, of $L$. This is done to avoid confusion with the dose, or energy, averages defined in eqns (19) and (20). 
and the second moments:

$$
\overline{y^{2}}=\overline{L^{2}} \overline{l^{2}} / \bar{l}^{2} \text {. }
$$

The dose average LET, $\bar{L}_{D}$, and the dose average lineal energy density, $\bar{y}_{D}$, are important quantities for practical applications:

$$
\bar{L}_{D}=\int_{0}^{\infty} L^{2} t(L) \mathrm{d} L / \int_{0}^{\infty} L t(L) \mathrm{d} L=\overline{L^{2}} / \bar{L}
$$

and if $f(y)$ is the distribution of $y$ :

$$
\bar{y}_{D}=\int_{0}^{\infty} y^{2} f(y) \mathrm{d} y / \int_{0}^{\infty} y f(y) \mathrm{d} y=\overline{y^{2}} / \bar{y}
$$

From eqns (18) to (20) one obtains the relation which connects $\bar{y}_{D}$ with $\bar{L}_{D}$ :

$$
\bar{y}_{D}=\bar{L}_{D} \overline{l^{2}} / \bar{l}^{2} \text {. }
$$

For a sphere of diameter $d$ one has according to eqn (3)

$$
\bar{l}=\frac{2}{3} d
$$

and

$$
\overline{l^{2}}=2 \int_{0}^{d} \frac{l^{3}}{d^{2}}=\frac{d^{2}}{2}
$$

and, therefore,

$$
\bar{y}_{D}=\frac{9}{8} \bar{L}_{D} \text {. }
$$

These formulae can be used to infer the track average and the dose average LET directly from the observed values $\bar{y}_{F}$ and $\bar{y}_{D}$; they also offer a convenient check of the numerical computations. For the example depicted in fig. 1 one obtains the values

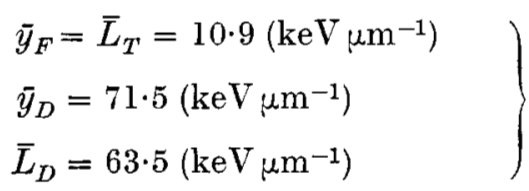

in agreement with eqns (17) and (24).

The relations for the moments given in this section are special cases of more general relations which have been derived earlier (Kellerer 1970), and which account for additional factors such as energy-loss straggling. The more general relations have to be used whenever the conditions discussed in section 1 are not met.

For mono-energetic neutron fields some of these conditions have been evaluated recently (Kellerer 197la). 


\section{Conclusions}

Rossi and Rosenzweig (1955) have derived a formula which permits the derivation of a LET-spectrum from an energy-deposition spectrum obtained with spherical proportional counters. Turner, Anderson, Birkhoff and Johnson (1970) have developed a Monte Carlo procedure to perform this analysis in the more general case of a non-spherical reference volume. They have also written a Monte Carlo program (Birkhoff, Turner, Anderson, Feola and Hamm 1970) to derive chord-length distributions for cylindrical regions. The solution described in the present paper can be used as an alternative to the established methods. It is analytical and, therefore, not limited in precision; it is also applicable to regions of arbitrary shape or to cases where one deals with regions of varying size. Convenient analytical expressions for chord-length distributions in cylinders have been derived recently (Kellerer 1971b). These distributions in conjunction with the solution discussed in this paper can be used for the analysis of measurements obtained in cylindrical proportional counters.

This paper is based on work performed under Contract AT-(30-1)-2740 for the U.S. Atomic Energy Commission.

\section{RÉSUMe}

Un algorithme pour l'analyse du transfert linéaire d'énergie

On décrit un algorithme pour la dérivation des distributions de LET de spectres des hauteurs d'impulsions, obtenues au moyen des compteurs proportionnels. La méthode se base sur la transformation de Fourier; elle est applicable aux compteurs proportionnels sphériques et non. sphériques. La relation est donnée entre la valeur moyenne d'énergie, $\bar{L}_{D}$, de LET, et la valeur moyenne d'énergie, $\bar{y}_{D}$, de l'événement.

\section{ZUSAMMENFASSUNG}

Ein Algorithmus für die Analyse des linearen Energieumsatzes (LET)

Man beschreibt einen Algorithmus für die Ableitung der LET-Verteilungen aus den mittels Proportionalzähler erhaltenen Impulsamplitudenspektra. Das Verfahren basiert auf der FourierTransformation; es ist sowohl für sphärische als auch für nichtsphärische Proportionalzähler geeignet. Es wird das Verhältnis zwischen dem Energiedurchschnittswert, $\bar{L}_{D}$, von LET, und dem Energiedurchschnittswert, $\bar{y}_{D}$, des Ereignisses angegeben.

\section{Резюме}

Альгоритм для анализа линейной передачи энергии

Дается описание алыгоритма для вывода распределений линейной передачи энергии из амплитудного спектра импульсов, полученного при помощи пропорциональных счетчиков. Метод основывается на преобразованиях Фурье; он применим для случая сферических, а также несферических пропорциональных счетчиков. Дается отношение между средней величиной энергии, $\bar{L}_{D}$, для ЛПЭ и средней величиной энергии, $\bar{y}_{D}$, события. 


\section{REFERENCES}

Bravati, M. H., Rossi, H. H., and Boer, E., 1965, Ann. Rep. on Research Project, AEC-2740-2, pp. 41-76.

Birkhoff, R. D., Turner, J. E., Axdersen, V. E., Feola, J. M., and Hamm, R. N., 1970, Health Phys., 18, 1.

Cooley, I. W., and Tukey, J. W., 1965, Maths Comput., 19, 297.

Feller, W., 1950, Probability Theory and Its Applications, Vol. I (New York: Wiley).

ICRU, 1971, Radiation Quantities and Units, (Washington DC: International Commission on Radiation Units and Measurements) Report 19.

Kellerer, A. M., 1970, in 'Microdosimetry', Proc. Second Symp. on Microdosimetry, ed. H. G. Ebert (Brussels: Euratom).

KELLERER, A. M., 197la, Radiat. Res., 47, 377.

Kellerer, A. M., 1971b, Radiat. Res., 47, 359.

Rossi, H. H., and RozenzweIG, W., 1955, Radiat. Res., $2,417$.

Ttrner, J. E., Anderson, V. E., Birkhoff, R. D., and Johrson, D. R., 1970, Health Phys., 18, 15.

Wilson, K. S. J., and Field, S. B., 1970, Phys. Med. Biol., 15, 657. 